\title{
Prioritizing chronic obstructive pulmonary disease (COPD) candidate genes in COPD-related networks
}

\author{
Yihua Zhang ${ }^{1, *}$, Wan Li ${ }^{1, *}$, Yuyan Feng ${ }^{1, *}$, Shanshan Guo ${ }^{1}$, Xilei Zhao ${ }^{1}$, Yahui Wang ${ }^{1}$, \\ Yuehan $\mathrm{He}^{1}$, Weiming $\mathrm{He}^{2}$ and Lina Chen ${ }^{1}$ \\ ${ }^{1}$ College of Bioinformatics Science and Technology, Harbin Medical University, Harbin, Heilongjiang Province, China \\ ${ }^{2}$ Institute of Opto-Electronics, Harbin Institute of Technology, Harbin, Heilongjiang Province, China \\ *These authors contributed equally to this work \\ Correspondence to: Lina Chen, email: chenlina@ems.hrbmu.edu.cn \\ Weiming He, email: hewm@hit.edu.cn
}

Keywords: chronic obstructive pulmonary disease, gene prioritization, metabolic network, protein-protein interaction network, functional information

Received: September 07, $2017 \quad$ Accepted: October 04, $2017 \quad$ Published: October 17, 2017

Copyright: Zhang et al. This is an open-access article distributed under the terms of the Creative Commons Attribution License 3.0 (CC BY 3.0), which permits unrestricted use, distribution, and reproduction in any medium, provided the original author and source are credited.

\section{ABSTRACT}

Chronic obstructive pulmonary disease (COPD) is a multi-factor disease, which could be caused by many factors, including disturbances of metabolism and proteinprotein interactions (PPIs). In this paper, a weighted COPD-related metabolic network and a weighted COPD-related PPI network were constructed base on COPD disease genes and functional information. Candidate genes in these weighted COPD-related networks were prioritized by making use of a gene prioritization method, respectively. Literature review and functional enrichment analysis of the top $\mathbf{1 0 0}$ genes in these two networks suggested the correlation of COPD and these genes. The performance of our gene prioritization method was superior to that of ToppGene and ToppNet for genes from the COPD-related metabolic network or the COPD-related PPI network after assessing using leave-one-out cross-validation, literature validation and functional enrichment analysis. The top-ranked genes prioritized from COPD-related metabolic and PPI networks could promote the better understanding about the molecular mechanism of this disease from different perspectives. The top 100 genes in COPDrelated metabolic network or COPD-related PPI network might be potential markers for the diagnosis and treatment of COPD.

\section{INTRODUCTION}

Chronic obstructive pulmonary disease (COPD) is the third leading cause of morbidity and mortality worldwide [1]. As a complex disease, COPD is caused by many factors, including smoking, advanced age, systemic inflammation, and especially disturbances of metabolism [2] and protein-protein interactions (PPIs). For example, glucose metabolism disturbances were more observed in COPD patients than in control individuals [3]. An elevated energy metabolism was also detected in COPD patients [4]. In the COPD pathogenesis, the interaction between CCR6 and its ligand CCL20 promotes the effect of dendritic cells [5]. The interaction of TPP1 with the
Sirtuin 1 complex could be disrupted by cigarette smoke. This caused reduced level of TPP1 on telomeres in lungs from COPD patients [6].

Molecular changes occurring in the process of complex diseases could be represented in terms of metabolic networks [7] and PPI networks, which have been used in many researches from various aspects. Shang et al. identified disease-related metabolites from a global metabolic network based on the assumption that the metabolites related to the same disease tend to be modularized in metabolic networks. Good performance and robustness were achieved for different disease classes, especially for respiratory diseases [8]. By integrating coexpression networks with metabolic networks, $\mathrm{Ni}$ et 
al. developed a computational method to predicted key enzyme-coding genes in both Parkinson's disease and Huntington's disease. These predicted metabolic genes might act as novel biomarkers the diagnosis and potential therapeutic treatments of these diseases [9]. Wang et al. identified 23 novel genes potentially related to infertility from a human PPI network based on previously validated infertility-related genes. The identified genes were strongly related to dysfunction of four main biological processes of fertility [10]. By integrating the gene expression profile data and PPI data, Huo et al. constructed two coexpression PPI networks in a coronary heart disease (CHD) state and a non-CHD state. They found that the treatment of CHD with Danshensu may be partly attributed to the regulation of immunization and blood circulation. Several potential therapeutic targets for CHD were also identified [11]. Integrating other information into networks could help to better reveal disease mechanisms. Blais et al. predicted biomarker changes in response to drugs by integrating transcriptomics data to metabolic networks for hepatocytes. Their results were validated with literature-based evidence and new experimental data [12]. Zeng et al. used a novel relevance measure to prioritize candidate disease genes based on a heterogeneous network integrating PPI and phenotype information. The 3-fold experiments showed that their methods were better than or similar to existing methods [13]. Transcriptome data were integrated to PPI networks of differentially expressed genes in peripheral blood mononuclear cells and pancreatic $\beta$-cellsto to identify key genes associated with Type 1 diabetes risk [14]. Many researches have found that genes with similar functions are more likely to be associated with similar diseases [15-17]. Therefore, it is necessary to further integrate functional information into disease-related networks to study the mechanism of diseases.

In this paper, two weighted COPD-related networks were constructed base on COPD disease genes and functional information. Candidate genes in each COPDrelated network were prioritized by making use of a gene prioritization method, respectively. The top-ranked genes in the COPD-related metabolic network or COPD-related PPI network could reflect the molecular mechanism of COPD and might be potential markers for its diagnosis and treatment.

\section{RESULTS}

Base on COPD disease genes, a COPD-related metabolic network and a COPD-related PPI network were constructed, respectively. Nodes and edges of these two COPD-related networks were weighted by integrating functional information. For genes in each COPD-related network, disease risk scores were calculated taking the transfer of disease risks into consideration.

\section{Gene prioritization}

COPD candidate genes were prioritized in each COPD-related network according to their risk scores in descending order (details in Methods). The top-ranked genes in each network had higher disease risk scores and were more associated with COPD. To further demonstrate the relationships between these genes and COPD, literature validation and functional enrichment analysis were applied for the top 100 genes in each COPD-related network.

For the top 100 genes in the COPD-related metabolic network, it was found that higher ranked genes were validated with higher proportion in literature. That is, $56 \%$ of the top 100 genes, $66 \%$ of the top 50 genes and $90 \%$ of the top 10 genes were associated with COPD in literature, such as CYP2E1 (Rank: 1), CYP2C9 (Rank: 4), NOS1 (Rank: 5) and CYP1B1 (Rank: 8). These associations have been explained in our previous work [18].

For top genes in the COPD-related PPI network, $61 \%$ of the top 100 genes and $72 \%$ of the top 50 genes have been validated by literature, though only $40 \%$ of the top 10 genes were validated to be associated with COPD by literature. COPD was independently associated with lower prevalences of EGFR (Rank: 2) mutations [19]. Human COPD lungs had decreased protein levels of CTNNB1 (Rank: 4), which was positively correlated with pulmonary function [20, 21]. The protein level of UBB (Rank: 7) was significantly different between control and COPD lung tissue by western analysis [22]. Higher SRC (Rank: 8) activation was measured in small airway epithelial cells from patients with COPD compared with healthy control subjects, which indicated that the activation of SRC promotes COPD-related processes [23].

Of the top 100 genes in two COPD-related networks, 11 genes were common (Figure 1), 8 of which have been validated by literature. For example, SOD1 was supposed to participate in the antioxidant defense of lungs in COPD patients, since its protein levels were found to be significantly higher in COPD patients than in those with no COPD [24]. Quantitative digital image analysis revealed increased cytoplasmic expression of FGF2 in bronchial epithelium and airway smooth muscle in COPD patients compared with controls [25]. Zanini et al. also found that FGF2 were significantly increased in COPD patients as compared to controls [26]. These common genes played important roles in COPD. It was speculated that unique genes of the top 100 genes prioritized from COPD-related metabolic and PPI networks could reflect the molecular mechanism of COPD from different perspectives. These common or unique genes could be involved in various COPD-related processes about metabolism or protein interactions.

Functions annotated by COPD disease genes were defined as COPD-related functions. 45 COPD-related 
functions were significantly enriched by the top 100 genes in the COPD-related metabolic network and the top 100 genes in the COPD-related PPI network (Benjamini adjusted $P$ value $<0.05$ ) (some are illustrated in Figure 1 and Figure 2), including "Angiogenesis" and "extracellular regions". The associations of these functions and COPD have been explained in our previous work [18].

153 GO functions were significantly enriched by the top 100 genes in the COPD-related metabolic network (Benjamini adjusted $P$ value $<0.05), 86$ (56.209\%) of which were COPD-related (some are illustrated in Figure 2). "Heme binding" was one of these COPDrelated functions, whose role in COPD has been described in our previous work [18]. The "organelle membrane"permeant iron chelator deferiprone could contribute to alleviate experimental COPD [27]. The "steroid metabolic process" was involved by downregulated genes screened from a dataset including three COPD samples and three normal samples [28].

The top 100 genes in the COPD-related PPI network were significantly enriched in $541 \mathrm{GO}$ functions (Benjamini adjusted $P$ value $<0.05)$. 223 (41.220\%) were
COPD-related functions (some are illustrated in Figure 2). "Transcription factor binding" site (TFBS) analysis confirmed that multiple COPD eQTL SNPs disrupted TFBS [29]. Dampening the "innate immune response" to smoking played a critical role in modifying pulmonary inflammation and lung remodeling, which might slow the progression of COPD [30]. Nasal epithelial cells are involved in many airway diseases, including asthma and COPD, through their "innate immune response" and interaction with immune and airway stromal cells [31]. Bush et al. found that genes important in "lung development" and early wheezing were implicated in COPD [32].

In COPD-related pathways that were annotated by COPD disease genes, 18 could be significantly enriched by the top 100 genes in the COPD-related metabolic network and the top 100 genes in the COPD-related PPI network (Benjamini adjusted $P$ value $<0.05$ ) (Some are illustrated in Figures 1 and 3). These pathways included both metabolic pathways and signaling pathways, which indicated their involvement in COPD. Through "regulation of the actin cytoskeleton" and up-regulation of integrin- $\beta 1$,

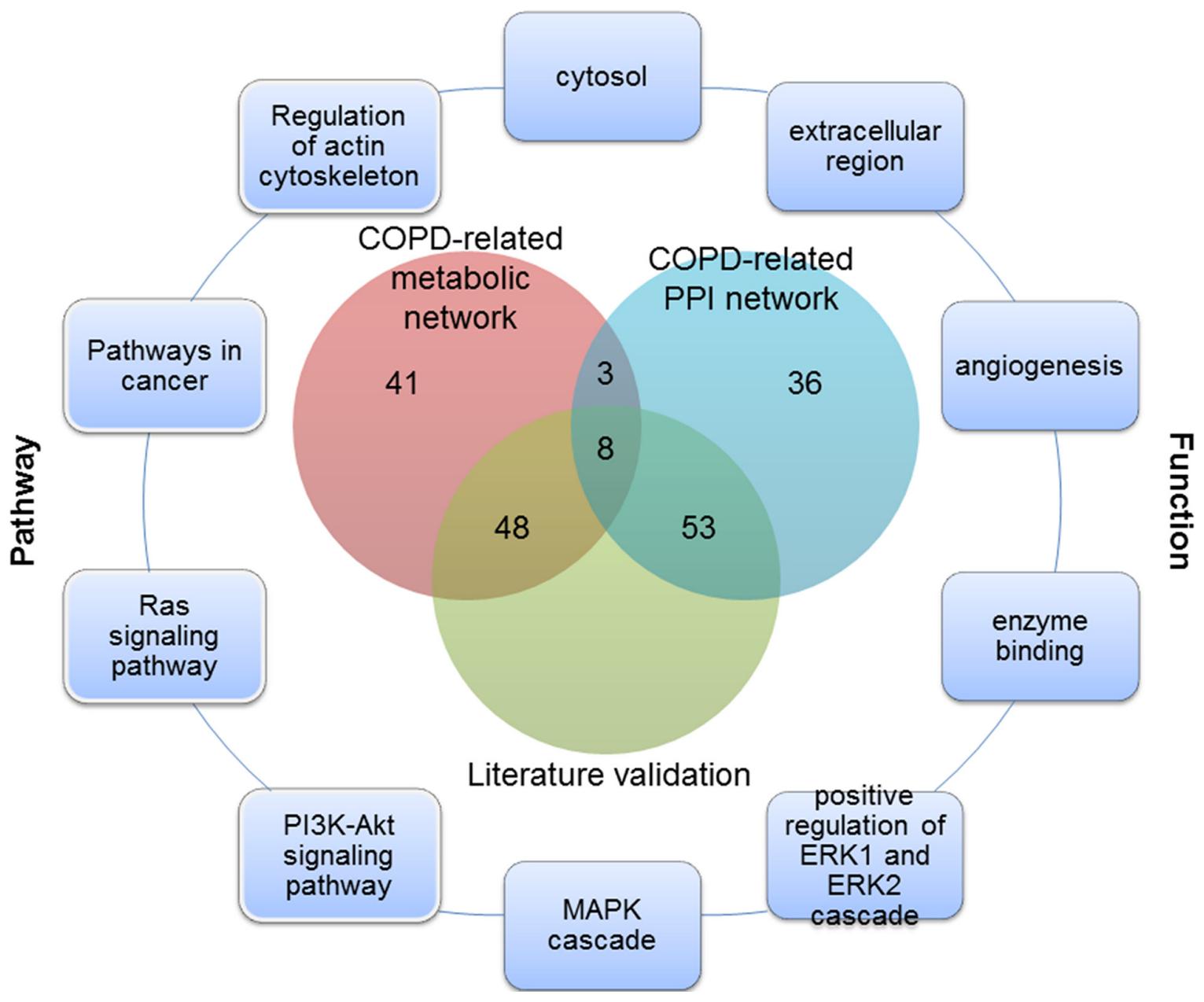

Figure 1: The overlap of the top 100 genes and literature validation from the COPD-related metabolic and PPI networks, and part of COPD-related functions and pathways for these 11 common genes. 
normal contractile function could be restored for COPD patients [33]. The "PI3K/Akt signaling pathway" was required for epithelial-mesenchymal transition in small airway fibrosis of COPD patients [34].

The top 100 genes in the COPD-related metabolic network were significantly enriched in 34 KEGG pathways (Benjamini adjusted $P$ value $<0.05), 32(94.118 \%)$ of which were COPD-related (Some are illustrated in Figure 3). Most of these pathways were "metabolic pathways", such as "Steroid hormone biosynthesis", "Metabolism of xenobiotics by Cytochrome P450" and "retinol metabolism". The associations of these pathways and COPD have been explained in our previous work [18].

104 KEGG pathways were significantly enriched by the top 100 genes in the COPD-related PPI network (Benjamini adjusted $P$ value $<0.05) .78(75 \%)$ were COPD-related pathways (Some are illustrated in Figure 3 ), most of which were signaling pathways. "T cell receptor signaling" molecules were down-regulated in COPD pulmonary CD8 cells [35]. In the development of "Non-small cell lung cancer", COPD and smoking played a vital role. Local progression and metastasis of "Non-small cell lung cancer" has been associated with the epithelial mesenchymal transition, which was implicated in COPD pathogenesis [36]. "Platelet activation" was a potential therapeutic target in patients with COPD aiming to reduce their risk of thrombosis or other cardiovascular events [37-39].

These results demonstrated the top-ranked genes in each COPD-related network were more associated with COPD, and could be enriched in COPD-related functions or pathways.

\section{Performance evaluation and comparison}

The performance of our gene prioritization method was assessed for each COPD-related network using leaveone-out cross-validation (LOOCV) (details in Methods). Then, our method was compared with ToppGene and ToppNet based on the area under the receiver operating characteristic (ROC) curve (AUC). ToppGene and ToppNet are two tools in the ToppGene Suite (https:// ToppGene.cchmc.org) [40] for prioritizing candidate genes based on a set of disease genes considering various factors, such as GO annotations and protein interactions. It was showed that AUCs of our gene prioritization method for both COPD-related networks (0.949 and 0.799) were higher than those of ToppGene (0.912 and 0.714) and ToppNet (0.854 and 0.687) (Figure 4).

The three methods were also compared on the validated proportion of their top 100 genes in literature. For genes from the COPD-related metabolic network,

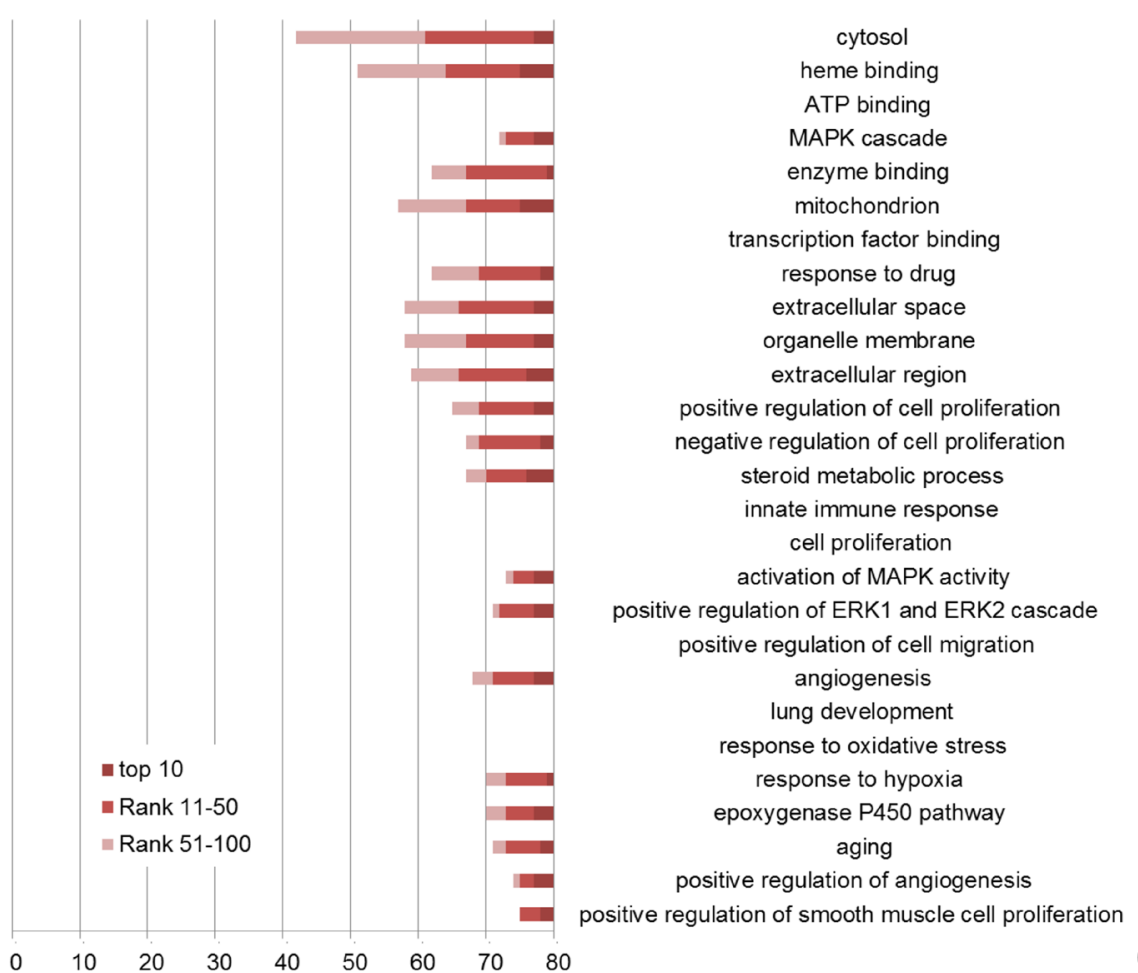

COPD-related metabolic network
Functions

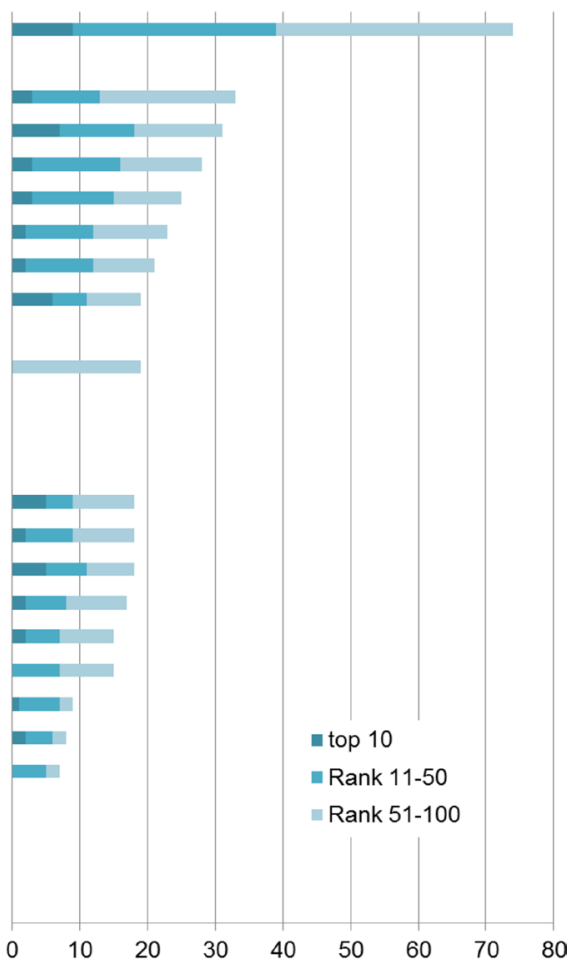

COPD-related PPI network

Figure 2: Some of COPD-related GO functions significantly enriched by the top 100 genes in the COPD-related metabolic network (left) and the top $\mathbf{1 0 0}$ genes in the COPD-related PPI network (right). GO functions (horizontal axis) were significantly enriched by the top 100 genes (the number in the vertical axis) using DAVID (Benjamini corrected $P$ value $<0.05$ ). 
the proportions for ToppGene and ToppNet have been described in our previous work (Supplementary Figure 1) [18], which were less than that for our gene prioritization method. For genes from the COPD-related PPI network, $47 \%$ of the top $100,42 \%$ of the top 50 , and $50 \%$ of the top 10 genes prioritized by ToppGene were validated, while $31 \%$ of the top $100,30 \%$ of the top 50 , and $30 \%$ of the top 10 genes prioritized by ToppNet were validated to be involved in COPD (Supplementary Figure 2). Most of these proportions were less than those of our gene prioritization method $(61 \%, 72 \%$ and $40 \%)$.

The performance of the three methods were further compared on enriched COPD-related function or pathway proportion of the top 100 genes employing functional enrichment analysis (Supplementary Table 1). For genes from the COPD-related metabolic network, the comparison of the numbers and proportions of COPD- related functions or pathways have been described in our previous work [18]. For genes from the COPD-related PPI network, the top 100 genes of our gene prioritization method could be enriched in more COPD-related functions or pathways than those of ToppGene and ToppNet, although the proportions were slightly smaller.

These results showed that the top-ranked genes of our gene prioritization method had better performance on AUC of LOOCV, literature validation and COPD-related function or pathway proportion. Thus, these genes were more associated with COPD than those of ToppGene and ToppNet for both COPD-related networks.

\section{DISCUSSION}

In this paper, COPD candidate genes were prioritized in two weighted COPD-related networks

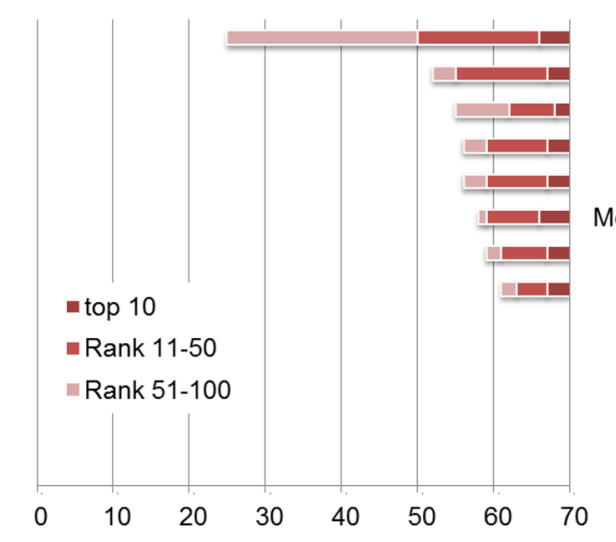

COPD-related metabolic network

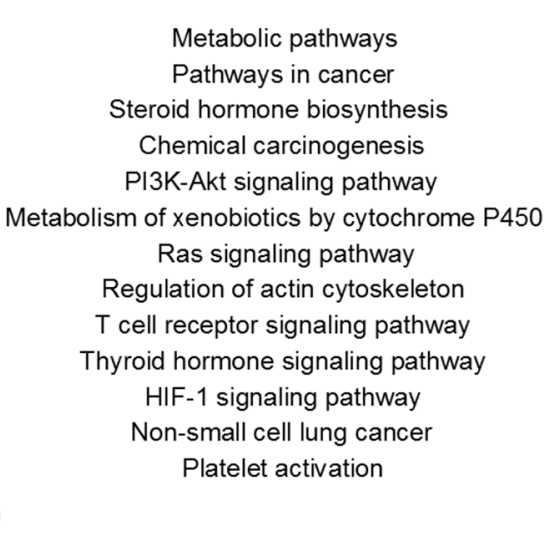

Pathways

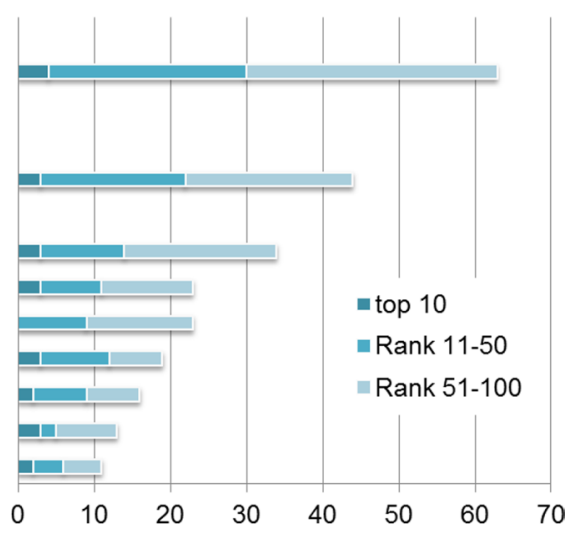

COPD-related PPI network

Figure 3: Some of COPD-related KEGG pathways significantly enriched by the top 100 genes in the COPD-related metabolic network (left) and the top $\mathbf{1 0 0}$ genes in the COPD-related PPI network (right). KEGG pathways (horizontal axis) were significantly enriched by the top 100 genes (the number in the vertical axis) using DAVID (Benjamini corrected $P$ value $<0.05$ ).
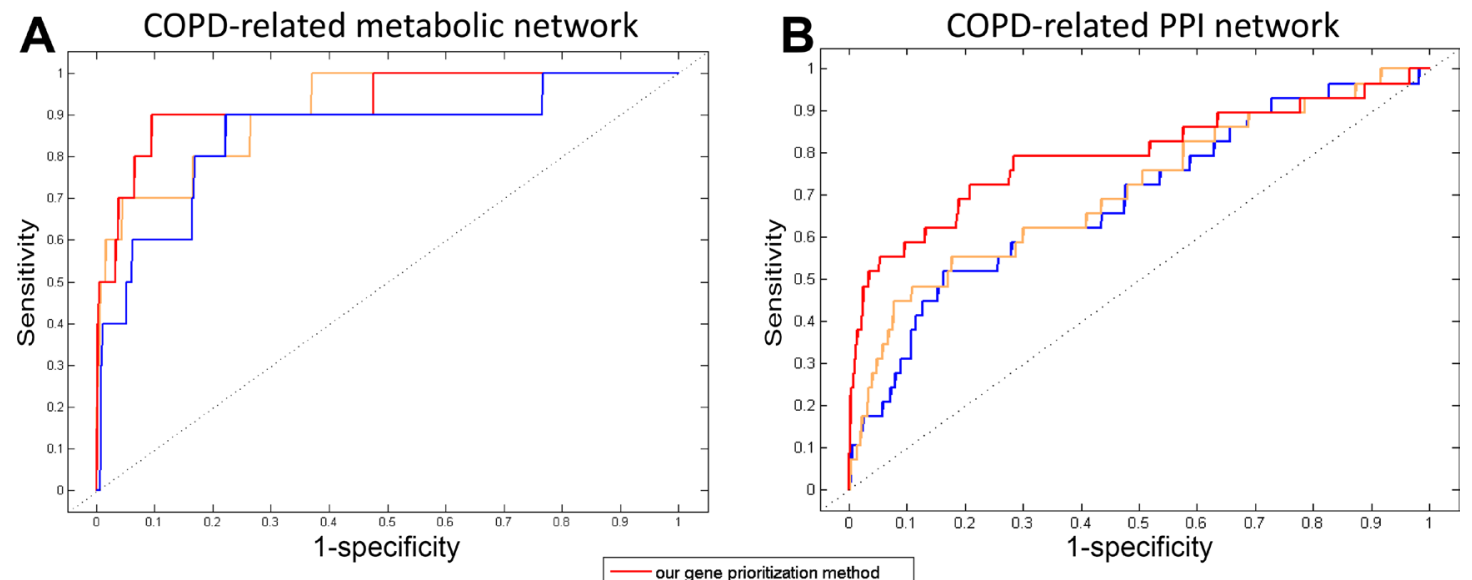

\begin{tabular}{|l|}
\hline - our gene prioritization method \\
ToppGene \\
- ToppNet
\end{tabular}

Figure 4: The ROC curves of our gene prioritization method, ToppGene and ToppNet for COPD-related (A) metabolic and (B) PPI networks. 
according to their risk scores by using a gene prioritization method, respectively. Literature validation and functional enrichment analysis were assessed for the top 100 genes from each COPD-related network. The performance of the gene prioritization method was superior to that of ToppGene and ToppNet on AUC of LOOCV, literature validation and COPD-related function or pathway proportion for their top 100 genes.

To further exhibit the classification performance of the top-ranked genes in two COPD-related networks, a support vector machine with linear kernel was employed to classify samples of a COPD-related expression profile GSE57148. The profile was obtained from Gene Expression Omnibus (GEO, https://www.ncbi.nlm.nih. gov/geo/) [41], which contained 98 COPD patients and 91 normal controls. The classification process was conducted for the top 10 (the same number as COPD disease genes in the COPD-related metabolic network), the top 29 (the same number as all COPD disease genes) and the top 100 genes in two COPD-related networks, respectively. Then the same classification process was conducted for 10 COPD disease genes in the COPD-related metabolic network and 29 COPD disease genes in the COPD-related PPI network (see Data). AUC was used to compare their classification performance (Table 1). It was showed that the classification performance of the top 10 genes in both COPD-related networks was better than that of 10 COPD disease genes. The classification performance of 29 COPD disease genes was better than that of the top 29 genes in the COPD-related metabolic network, while that of the top 29 genes in the COPD-related PPI network was even better. The top 100 genes in two COPD-related networks could both classify samples with good performance.

The performance of the top 100 genes in the COPDrelated metabolic network was better on the numbers and proportions of enriched functions or pathways and AUC of LOOCV than those from the COPD-related PPI network, while the performance of the top 100 genes in the COPDrelated PPI network was better on literature validation and the classification performance than those from the COPDrelated metabolic network. These results indicated that the top-ranked genes prioritized from these two COPDrelated networks could reflect the molecular mechanism of COPD from different perspectives by participating in various COPD-related processes about metabolism or protein interactions.

To conclude, COPD candidate genes were prioritized in COPD-related networks using the gene prioritization method. The correlation of the top 100 genes and COPD was validated by literature and functional enrichment analysis. Compared with ToppGene and ToppNet, our gene prioritization method had better performance. The top-ranked genes prioritized from COPD-related metabolic and PPI networks could promote the better understanding about the molecular mechanism of this disease from different perspectives. The top 100 genes in either COPD-related network might be potential markers for the diagnosis and treatment of COPD.

\section{MATERIALS AND METHODS}

\section{Data}

COPD disease genes were obtained from databases and literature, including Online Mendelian Inheritance in Man (OMIM, https://www.omim.org/) [42], the Disease Ontology (DO, http://disease-ontology.org/) [43], Phenotype-Genotype Integrator (PheGenI) (https://www. ncbi.nlm.nih.gov/gap/phegeni) [44], DISEASES (http:// diseases.jensenlab.org/) [45] and Menche's research [46]. A total of 29 COPD disease genes were collected for further analysis.

Gene functional information was extracted as all annotation terms for human genes in three ontologies, i.e. biological processes, molecular functions and cellular components, from Gene Ontology (GO, http://www. geneontology.org/) [47].

\section{Construction of weighted COPD-related networks}

Based on these COPD disease genes, two weighted COPD-related networks were constructed. One was a COPD-related metabolic network, which was built using COPD disease genes and their direct interactors extracted from an integrated human metabolic network as described in our previous work [18]. The COPD-related metabolic network contained 1361 genes and their 6601 interactions, 10 of which were COPD disease genes, and others were candidate genes. The other was a COPD-related PPI network, which was retrieved using COPD disease gene products and their interacting partners from the STRING database (http://string-db.org/) [48]. The COPD-related PPI network was comprised of 7791 interactions between 3740 proteins (gene products). All of 29 COPD disease genes were in the network, and other genes were candidate ones.

Weights for genes and interactions (nodes and edges of these COPD-related networks) were calculated by integrating functional information as in our previous work [18].

\section{Prioritization of candidate genes}

To prioritize candidate genes in each COPD-related network, disease risk score of each gene was obtained taking the transfer of disease risks into consideration, respectively:

$$
D^{(i=1)}=(1-\beta) Q D^{(i)}+\beta D^{(0)}
$$

where $D(i)$ is the vector of risk scores of all genes at step $i$, and $\beta \in(0,1)$ is a parameter to measure the 
Table 1: The classification performance (AUC) of top 10, 29 and 100 genes in both COPD-related networks, and of 10 and 29 COPD disease genes

\begin{tabular}{lccc}
\hline & $\mathbf{1 0}^{\mathbf{a}}$ & $\mathbf{2 9}^{\mathbf{a}}$ & $\mathbf{1 0 0}^{\mathbf{a}}$ \\
\hline COPD- related metabolic network & 0.729 & 0.810 & 0.789 \\
COPD- related PPI network & 0.853 & 0.896 & 0.932 \\
COPD disease genes & 0.725 & 0.837 & - \\
\hline
\end{tabular}

${ }^{\text {a }}$ The number of top-ranked genes used to classify samples.

importance between genes and interactions. After assessing the performance using $\beta=0.1,0.2, \cdots, 0.9, \beta=0.1$ was chosen as the optimal parameter. $Q$ is the disease risk transition probability matrix, whose element $q(g \mid h)$, the disease risk going from gene $h$ to gene $g$, was defined as

$$
q(g \mid h)=\frac{w_{(h, g)}}{\sum_{l \in \text { neighbor }(h)} w_{(l, g)}}
$$

where $w_{(h, g)}$ is the interaction weight between interacting genes $h$ and $g$, and neighbou( $h$ ) is the set of genes that interact with gene $h . D^{(0)}$ is the vector of initial disease risk scores for all genes. Each element of $D^{(0)}$, i.e. score $\mathrm{d}^{g(0)}$ for gene $g$ in each COPD-related network, was calculated as follows:

$$
d_{g}{ }^{(0)}=\frac{w_{g}}{\sum_{m \in \text { COPD-related metabolic/PPI network }} w_{m}}
$$

The process was carried out until the difference between $D^{(i)}$ and $D^{(i+1)}$ was less than a threshold, $10^{-9}$. Candidate genes from each COPD-related network were prioritized according to their risk scores in descending order.

To examine the association between the topranked genes and COPD, literature validation was performed for the top 100 genes in each COPD-related network in literature of PubMed (http://www.ncbi.nlm. nih.gov/pubmed). Then, functional enrichment analysis was applied for the top 100 genes using the Functional Annotation Tool in the Database for Annotation, Visualization and Integrated Discovery (DAVID, http:// david.abcc.ncifcrf.gov/) v6.8 [49, 50]. GO functions and KEGG pathways with adjusted $P$ value (Benjamini) less than 0.05 were considered significant.

\section{Evaluation and comparison of the performance}

LOOCV was carried out to assess the performance of our gene prioritization method as described our previous work [18]. The ROC curves were plotted and AUC was computed based on the ranks of test genes. These results were compared with those of ToppGene and ToppNet using genes from COPD-related metabolic and PPI networks, respectively.

To compare with the top 100 genes of our gene prioritization method, literature validation and functional enrichment analysis for the top 100 genes prioritized by ToppGene and ToppNet were also performed.

\section{Author contributions}

LC and WH conceived and designed the study. YZ and SG collected data. YZ, WL and YF performed the analysis. YZ and WL wrote the paper. SG, XZ, YW, YH and $\mathrm{LC}$ reviewed and edited the manuscript. All authors read and approved the manuscript.

\section{CONFLICTS OF INTEREST}

The authors declare no conflict of interest.

\section{FUNDING}

This work was supported in part by the National Natural Science Foundation of China (Grant No. 61702141 and 61272388); the Health and Family Planning Commission Scientific Research Subject of Heilongjiang Province (Grant No. 2016-203); the Innovative Scientific Research Funding Project of Harbin Medical University (2017JCZX46); and the Harbin Applied Technology Research and Development Project (Grant No. 2016RQQXJ105).

\section{REFERENCES}

1. Vestbo J, Hurd SS, Agusti AG, Jones PW, Vogelmeier C, Anzueto A, Barnes PJ, Fabbri LM, Martinez FJ, Nishimura M, Stockley RA, Sin DD, Rodriguez-Roisin R. Global strategy for the diagnosis, management, and prevention of chronic obstructive pulmonary disease: GOLD executive summary. Am J Respir Crit Care Med. 2013; 187:347-65. https://doi.org/10.1164/rccm.201204-0596PP.

2. Breyer MK, Spruit MA, Hanson CK, Franssen FM, Vanfleteren LE, Groenen MT, Bruijnzeel PL, Wouters EF, Rutten EP. Prevalence of metabolic syndrome in COPD patients and its consequences. PLoS One. 2014; 9:e98013. https://doi.org/10.1371/journal.pone.0098013.

3. Mirrakhimov AE. Chronic obstructive pulmonary disease and glucose metabolism: a bitter sweet symphony. Cardiovasc Diabetol. 2012; 11:132. https://doi.org/10.1186/1475-284011-132.

4. Schols AM. Nutritional and metabolic modulation in chronic obstructive pulmonary disease management. Eur Respir J Suppl. 2003; 46:81s-6s.

5. Sun D, Ouyang Y, Gu Y, Liu X. Cigarette smoke-induced chronic obstructive pulmonary disease is attenuated 
by CCL20-blocker: a rat model. Croat Med J. 2016; 57:363-70.

6. Ahmad T, Sundar IK, Tormos AM, Lerner CA, Gerloff J, Yao H, Rahman I. Shelterin Telomere Protection Protein 1 Reduction Causes Telomere Attrition and Cellular Senescence via Sirtuin 1 Deacetylase in Chronic Obstructive Pulmonary Disease. Am J Respir Cell Mol Biol. 2017; 56:38-49. https://doi.org/10.1165/rcmb.201601980 C.

7. Diez D, Agusti A, Wheelock CE. Network analysis in the investigation of chronic respiratory diseases. From basics to application. Am J Respir Crit Care Med. 2014; 190:981-8. https://doi.org/10.1164/rccm.201403-0421PP.

8. Shang D, Li C, Yao Q, Yang H, Xu Y, Han J, Li J, Su F, Zhang Y, Zhang C, Li D, Li X. Prioritizing candidate disease metabolites based on global functional relationships between metabolites in the context of metabolic pathways. PLoS One. 2014; 9:e104934. https://doi.org/10.1371/ journal.pone.0104934.

9. Ni Q, Su X, Chen J, Tian W. Prediction of Metabolic Gene Biomarkers for Neurodegenerative Disease by an Integrated Network-Based Approach. Biomed Res Int. 2015; 2015:432012. https://doi.org/10.1155/2015/432012.

10. Wang S, Huang G, Hu Q, Zou Q. A network-based method for the identification of putative genes related to infertility. Biochim Biophys Acta. 2016; 1860:2716-24. https://doi. org/10.1016/j.bbagen.2016.04.010.

11. Huo M, Wang Z, Wu D, Zhang Y, Qiao Y. Using Coexpression Protein Interaction Network Analysis to Identify Mechanisms of Danshensu Affecting Patients with Coronary Heart Disease. Int J Mol Sci. 2017; 18. https://doi. org/10.3390/ijms18061298.

12. Blais EM, Rawls KD, Dougherty BV, Li ZI, Kolling GL, Ye P, Wallqvist A, Papin JA. Reconciled rat and human metabolic networks for comparative toxicogenomics and biomarker predictions. Nat Commun. 2017; 8:14250. https://doi.org/10.1038/ncomms14250.

13. Zeng X, Liao Y, Liu Y, Zou Q. Prediction and Validation of Disease Genes Using HeteSim Scores. IEEE/ACM Trans Comput Biol Bioinform. 2017; 14:687-95. https://doi. org/10.1109/TCBB.2016.2520947.

14. Safari-Alighiarloo N, Taghizadeh M, Tabatabaei SM, Shahsavari S, Namaki S, Khodakarim S, Rezaei-Tavirani M. Identification of new key genes for type 1 diabetes through construction and analysis of protein-protein interaction networks based on blood and pancreatic islet transcriptomes. J Diabetes. 2017; 9:764-77. https://doi. org/10.1111/1753-0407.12483.

15. Li P, Guo M, Wang C, Liu X, Zou Q. An overview of SNP interactions in genome-wide association studies. Brief Funct Genomics. 2015; 14:143-55. https://doi.org/10.1093/ bfgp/elu036.

16. Zou Q, Li J, Song L, Zeng X, Wang G. Similarity computation strategies in the microRNA-disease network: a survey. Brief Funct Genomics. 2016; 15:55-64. https:// doi.org/10.1093/bfgp/elv024.

17. Liu Y, Zeng X, He Z, Zou Q. Inferring microRNAdisease associations by random walk on a heterogeneous network with multiple data sources. IEEE/ACM Trans Comput Biol Bioinform. 2016. https://doi.org/10.1109/ TCBB.2016.2550432.

18. Wang X, Li W, Zhang Y, Feng Y, Zhao X, He Y, Zhang J, Chen L. Chronic obstructive pulmonary disease candidate gene prioritization based on metabolic networks and functional information. PLoS One. 2017; 12:e0184299. https://doi.org/10.1371/journal.pone.0184299.

19. Lim JU, Yeo CD, Rhee CK, Kim YH, Park CK, Kim JS, Kim JW, Lee SH, Kim SJ, Yoon HK, Kim TJ, Lee KY. Chronic Obstructive Pulmonary Disease-Related Non-Small-Cell Lung Cancer Exhibits a Low Prevalence of EGFR and ALK Driver Mutations. PLoS One. 2015; 10:e0142306. https:// doi.org/10.1371/journal.pone.0142306.

20. Jiang Z, Lao T, Qiu W, Polverino F, Gupta K, Guo F, Mancini JD, Naing ZZ, Cho MH, Castaldi PJ, Sun Y, Yu J, LauchoContreras ME, et al. A Chronic Obstructive Pulmonary Disease Susceptibility Gene, FAM13A, Regulates Protein Stability of beta-Catenin. Am J Respir Crit Care Med. 2016; 194:185-97. https://doi.org/10.1164/rccm.201505-0999OC.

21. Liu QX, Liu XS, Ni W, Chen SX, Xu YJ. [Expression of beta-catenin in human pulmonary tissues of smokers with and without chronic obstructive pulmonary disease]. [Article in Chinese]. Zhonghua Jie $\mathrm{He} \mathrm{He} \mathrm{Hu} \mathrm{Xi} \mathrm{Za}$ Zhi. 2012; 35:828-32.

22. Stepaniants S, Wang IM, Boie Y, Mortimer J, Kennedy B, Elliott M, Hayashi S, Luo H, Wong J, Loy L, Coulter S, Roberts CJ, Hogg JC, et al. Genes related to emphysema are enriched for ubiquitination pathways. BMC Pulm Med. 2014; 14:187. https://doi.org/10.1186/1471-2466-14-187.

23. Geraghty P, Hardigan A, Foronjy RF. Cigarette smoke activates the proto-oncogene c-src to promote airway inflammation and lung tissue destruction. Am J Respir Cell Mol Biol. 2014; 50:559-70. https://doi.org/10.1165/ rcmb.2013-02580C.

24. Mateu-Jimenez M, Sanchez-Font A, Rodriguez-Fuster A, Aguilo R, Pijuan L, Fermoselle C, Gea J, Curull V, Barreiro E. Redox Imbalance in Lung Cancer of Patients with Underlying Chronic Respiratory Conditions. Mol Med. 2016. https://doi.org/10.2119/molmed.2015.00199.

25. Kranenburg AR, Willems-Widyastuti A, Mooi WJ, Saxena PR, Sterk PJ, de Boer WI, Sharma HS. Chronic obstructive pulmonary disease is associated with enhanced bronchial expression of FGF-1, FGF-2, and FGFR-1. J Pathol. 2005; 206:28-38. https://doi.org/10.1002/path.1748.

26. Zanini A, Spanevello A, Baraldo S, Majori M, Della Patrona S, Gumiero F, Aiello M, Olivieri D, Saetta M, Chetta A. Decreased maturation of dendritic cells in the central airways of COPD patients is associated with VEGF, TGFbeta and vascularity. Respiration. 2014; 87:234-42. https:// doi.org/10.1159/000356749. 
27. Cloonan SM, Glass K, Laucho-Contreras ME, Bhashyam AR, Cervo M, Pabon MA, Konrad C, Polverino F, Siempos II, Perez E, Mizumura K, Ghosh MC, Parameswaran H, et al. Mitochondrial iron chelation ameliorates cigarette smoke-induced bronchitis and emphysema in mice. Nat Med. 2016; 22:163-74. https://doi.org/10.1038/nm.4021.

28. Wei L, Xu D, Qian Y, Huang G, Ma W, Liu F, Shen Y, Wang Z, Li L, Zhang S, Chen Y. Comprehensive analysis of geneexpression profile in chronic obstructive pulmonary disease. Int J Chron Obstruct Pulmon Dis. 2015; 10:1103-9. https:// doi.org/10.2147/COPD.S68570.

29. Castaldi PJ, Cho MH, Zhou X, Qiu W, McGeachie M, Celli B, Bakke P, Gulsvik A, Lomas DA, Crapo JD, Beaty TH, Rennard S, Harshfield B, et al. Genetic control of gene expression at novel and established chronic obstructive pulmonary disease loci. Hum Mol Genet. 2015; 24:1200-10. https://doi.org/10.1093/hmg/ddu525.

30. Young RP, Hopkins RJ. The Mevalonate Pathway and Innate Immune Hyper-Responsiveness in the Pathogenesis of COPD and Lung Cancer: Potential for Chemoprevention. Curr Mol Pharmacol. 2017; 10:46-59. https://doi.org/10.21 74/1874467209666160112130016.

31. Ulm A, Mayhew CN, Debley J, Khurana Hershey GK, Ji H. Cultivate Primary Nasal Epithelial Cells from Children and Reprogram into Induced Pluripotent Stem Cells. J Vis Exp. 2016. https://doi.org/10.3791/53814.

32. Bush A. Lung Development and Aging. Ann Am Thorac Soc. 2016; 13:S438-S46. https://doi.org/10.1513/AnnalsATS.201602-112AW.

33. Campbell JD, McDonough JE, Zeskind JE, Hackett TL, Pechkovsky DV, Brandsma CA, Suzuki M, Gosselink JV, Liu G, Alekseyev YO, Xiao J, Zhang X, Hayashi S, et al. A gene expression signature of emphysema-related lung destruction and its reversal by the tripeptide GHK. Genome Med. 2012; 4:67. https://doi.org/10.1186/gm367.

34. Wang Q, Wang Y, Zhang Y, Xiao W. The role of uPAR in epithelial-mesenchymal transition in small airway epithelium of patients with chronic obstructive pulmonary disease. Respir Res. 2013; 14:67. https://doi. org/10.1186/1465-9921-14-67.

35. Grundy S, Plumb J, Lea S, Kaur M, Ray D, Singh D. Down regulation of $\mathrm{T}$ cell receptor expression in COPD pulmonary CD8 cells. PLoS One. 2013; 8:e71629. https:// doi.org/10.1371/journal.pone.0071629.

36. Mahmood MQ, Ward C, Muller HK, Sohal SS, Walters EH. Epithelial mesenchymal transition (EMT) and non-small cell lung cancer (NSCLC): a mutual association with airway disease. Med Oncol. 2017; 34:45. https://doi.org/10.1007/ s12032-017-0900-y.

37. Malerba M, Nardin M, Radaeli A, Montuschi P, Carpagnano GE, Clini E. The potential role of endothelial dysfunction and platelet activation in the development of thrombotic risk in COPD patients. Expert Rev Hematol. 2017:1-12. https://doi.org/10.1080/17474086.2017.1353416.
38. Makhlouf HA, Sadek SH, Nafady AA. Platelet function in diabetic and non-diabetic patients with chronic obstructive pulmonary disease: A case control study. Clin Respir J. 2016. https://doi.org/10.1111/crj.12477.

39. Malerba M, Olivini A, Radaeli A, Ricciardolo FL, Clini E. Platelet activation and cardiovascular comorbidities in patients with chronic obstructive pulmonary disease. Curr Med Res Opin. 2016; 32:885-91. https://doi.org/10.1185/0 3007995.2016.1149054.

40. Chen J, Bardes EE, Aronow BJ, Jegga AG. ToppGene Suite for gene list enrichment analysis and candidate gene prioritization. Nucleic Acids Res. 2009; 37:W305-11. https://doi.org/10.1093/nar/gkp427.

41. Barrett T, Wilhite SE, Ledoux P, Evangelista C, Kim IF, Tomashevsky M, Marshall KA, Phillippy KH, Sherman PM, Holko M, Yefanov A, Lee H, Zhang N, et al. NCBI GEO: archive for functional genomics data sets--update. Nucleic Acids Res. 2013; 41:D991-5. https://doi.org/10.1093/nar/ gks1193.

42. Amberger JS, Bocchini CA, Schiettecatte F, Scott AF, Hamosh A. OMIM.org: Online Mendelian Inheritance in Man (OMIM(R)), an online catalog of human genes and genetic disorders. Nucleic Acids Res. 2015; 43:D789-98. https://doi.org/10.1093/nar/gku1205.

43. Kibbe WA, Arze C, Felix V, Mitraka E, Bolton E, Fu G, Mungall CJ, Binder JX, Malone J, Vasant D, Parkinson H, Schriml LM. Disease Ontology 2015 update: an expanded and updated database of human diseases for linking biomedical knowledge through disease data. Nucleic Acids Res. 2015; 43:D1071-8. https://doi.org/10.1093/nar/ gku1011.

44. Ramos EM, Hoffman D, Junkins HA, Maglott D, Phan L, Sherry ST, Feolo M, Hindorff LA. Phenotype-Genotype Integrator (PheGenI): synthesizing genome-wide association study (GWAS) data with existing genomic resources. Eur J Hum Genet. 2014; 22:144-7. https://doi. org/10.1038/ejhg.2013.96.

45. Pletscher-Frankild S, Palleja A, Tsafou K, Binder JX, Jensen LJ. DISEASES: text mining and data integration of diseasegene associations. Methods. 2015; 74:83-9. https://doi. org/10.1016/j.ymeth.2014.11.020.

46. Menche J, Sharma A, Kitsak M, Ghiassian SD, Vidal M, Loscalzo J, Barabasi AL. Disease networks. Uncovering disease-disease relationships through the incomplete interactome. Science. 2015; 347:1257601. https://doi. org/10.1126/science. 1257601 .

47. Gene Ontology Consortium. going forward. Nucleic Acids Res. 2015; 43:D1049-56. https://doi.org/10.1093/nar/ gku1179.

48. Szklarczyk D, Franceschini A, Wyder S, Forslund K, Heller D, Huerta-Cepas J, Simonovic M, Roth A, Santos A, Tsafou KP, Kuhn M, Bork P, Jensen LJ, et al. STRING v10: protein-protein interaction networks, integrated over the tree of life. Nucleic Acids Res. 2015; 43:D447-52. https:// doi.org/10.1093/nar/gku1003. 
49. Huang da W, Sherman BT, Lempicki RA. Bioinformatics enrichment tools: paths toward the comprehensive functional analysis of large gene lists. Nucleic Acids Res. 2009; 37:1-13. https://doi.org/10.1093/nar/gkn923.
50. Huang da W, Sherman BT, Lempicki RA. Systematic and integrative analysis of large gene lists using DAVID bioinformatics resources. Nat Protoc. 2009; 4:44-57. https://doi.org/10.1038/nprot.2008.211. 